\title{
Ketaksamaan Hadamard pada Fungsi Konveks
}

\author{
Rivanxander Irawan ${ }^{1}$, Eridani $^{2}$ \& Abdulloh Jaelani ${ }^{3}$ \\ 1,2,3 Department of Mathematics, Faculty of Science and Technology, Universitas Airlangga \\ ${ }^{2}$ Corresponding author: eridani.dinadewi@gmail.com
}

\begin{abstract}
Pada penelitian ini, akan dibahas sifat-sifat fungsi gamma dan hubungannya dengan fungsi konveks. Selanjutnya akan ditinjau bentuk ketaksamaan Hadamard pada fungsi konveks. Fungsi konveks adalah fungsi dengan sifat garis yang menghubungkan dua titik di kurvanya akan selalu di atas kurva tersebut. Berdasarkan hasil pembahasan, didapatkan fakta bahwa fungsi gamma merupakan fungsi yang bersifat log konveks. Selain itu, diperoleh bentuk umum ketaksamaan Hadamard untuk fungsi $p$-konveks dengan $p>0$.
\end{abstract}

Keywords: fungsi konveks, fungsi p-konveks, fungsi Gamma, ketaksamaan hadamard.

\section{Pendahuluan}

Fungsi Konveks adalah salah satu topik dalam matematika yang penerapannya dalam bidang analisis dan statistika cukup luas. Salah satunya adalah untuk menjamin bahwa turunan kedua suatu fungsi bernilai nonnegatif jika fungsi tersebut adalah fungsi konveks dan mempunyai turunan kedua. Fungsi konveks pertama kali diperkenalkan oleh Holder, Stolz dan Hadamard pada tahun 1889-1893 dan terus menerus mengalami perkembangan setelah munculnya penelitian Jensen pada tahun 1905 yang berjudul "Sur les functions convexes et les inegalites entre les valeurs moyennes". Banyak teorema yang merupakan aplikasi dari fungsi konveks muncul setelah penelitian yang dilakukan Jensen tersebut [1]. Secara umum, sebuah fungsi disebut fungsi konveks jika berlaku :

$$
f\left((1-t) x_{1}+t x_{2}\right) \leq(1-t) f\left(x_{1}\right)+t f\left(x_{2}\right)
$$

dengan $t \in[0,1]$ dan $\mathrm{x}_{1}, \mathrm{x}_{2}$ sebarang anggota domain $\mathrm{f}$. Adapun fungsi f dikatakan fungsi konkaf jika fungsi $-\mathrm{f}$ adalah fungsi konveks. Secara geometris, fungsi konveks adalah fungsi dengan sifat garis yang menghubungkan $\left(\mathrm{x}_{1}, \mathrm{f}\left(\mathrm{x}_{1}\right)\right)$ dan $\left(\mathrm{x}_{2}, \mathrm{f}\left(\mathrm{x}_{2}\right)\right)$ selalu berada diatas grafik fungsi $\mathrm{f}$. Sebuah fungsi konveks tidak selalu mempunyai turunan di setiap titik. Sebagai contoh, $\mathrm{f}(\mathrm{x})=|\mathrm{x}|$ merupakan fungsi konveks pada $\mathbb{R}$ tetapi tidak mempunyai turunan di 0 [2].

Salah satu contoh aplikasi dari fungsi konveks adalah ketaksamaan Hadamard. Pada 1893, J.Hadamard menemukan ketaksamaan dasar integral pada fungsi konveks yaitu nilai f di median domainnya kurang dari atau sama dengan nilai rerata $f$, serta nilai rerata 
$\mathrm{f}$ kurang dari atau sama dengan nilai median $\mathrm{f}$. Hadamard membuktikan ketaksamaannya pada fungsi konveks yang kemudian mengalami perkembangan pada tahun-tahun berikutnya. Ketaksamaan Hadamard berperan penting pada suatu fungsi karena secara tak langsung ketaksamaan Hadamard dapat menaksir nilai rerata suatu fungsi, salah satunya adalah fungsi gamma [1]. Fungsi gamma adalah fungsi yang cukup istimewa karena sering terdapat pada persamaan-persamaan peluang dan statistika. Selain itu, fungsi gamma juga banyak digunakan untuk menyelesaikan integral-integral khusus yang tidak dapat diselesaikan secara analitik dan beberapa permasalahan di bidang fisika atau teknik. L.Euler (1707-1783) mendefinisikan fungsi gamma sebagai :

$$
\Gamma(n)=\int_{0}^{\infty} e^{-t} t^{n-1} d t
$$

dengan $n>0$. Adapun fungsi digamma adalah hasil turunan pertama dari fungsi gamma yaitu :

$$
\psi(\mathrm{z})=\frac{\mathrm{d}[\ln \Gamma(\mathrm{z})]}{\mathrm{dz}}=\frac{\Gamma^{\prime}(\mathrm{z})}{\Gamma(\mathrm{z})}
$$

dengan $\mathrm{z}>0[3]$.

Pada tahun 2007, Zhang dan Wan melakukan kajian tentang fungsi konveks dan memperkenalkan notasi p-konveks yang kemudian didefinisikan kembali dan dikembangkan oleh Iscan. Iscan mendefinisikan fungsi p-konveks sebagai fungsi yang bersifat :

$$
f\left(\left[t x^{p}+(1-t) y^{p}\right]^{\frac{1}{p}}\right) \leq t f(x)+(1-t) f(y),
$$

dengan $\mathrm{t} \in[0,1], \mathrm{p}>0$ dan $\mathrm{x}, \mathrm{y}$ sebarang anggota domain $\mathrm{f}$. Untuk $\mathrm{p}=1$ maka fungsi 1-konveks yang dimaksud adalah fungsi konveks klasik. Untuk $\mathrm{p}=2$ maka fungsi 2konveks yang dimaksud adalah fungsi yang bersifat

$$
f\left(\left[t x^{2}+(1-t) y^{2}\right]^{\frac{1}{2}}\right) \leq t f(x)+(1-t) f(y)[4] .
$$

Definisi[1] Misalkan $\mathrm{I} \subseteq \mathbb{R}$ dan $\mathrm{f}: \mathrm{I} \rightarrow \mathbb{R}$. Fungsi f dikatakan konveks jika :

$$
f(\lambda x+(1-\lambda) y) \leq \lambda f(x)+(1-\lambda) f(y),
$$

dengan $\mathrm{x}, \mathrm{y} \in \mathrm{I}$ dan $\lambda \in[0,1]$. Lebih jauh jika $-\mathrm{f}: \mathrm{I} \rightarrow \mathbb{R}$ konveks maka $\mathrm{f}: \mathrm{I} \rightarrow \mathbb{R}$ adalah fungsi konkaf. 
Untuk $\lambda=\frac{1}{2}$ maka akan diperoleh $f\left(\frac{x+y}{2}\right) \leq \frac{f(x)+f(y)}{2}$. Selanjutnya jika f memenuhi ketaksamaan tersebut maka f disebut sebagai fungsi J-Konveks.

Teorema[1] Misalkan $\mathrm{f}$ Konveks di $\mathrm{I}=[\mathrm{a}, \mathrm{b}] \subseteq \mathbb{R}$. untuk sebarang titik $\mathrm{x}_{1}, \ldots, \mathrm{x}_{\mathrm{n}} \in \mathrm{I}$ dan sebarang bilangan $r_{1}, \ldots, r_{n} \in Q^{+}$yang bersifat $r_{1}+\cdots+r_{n}=1$ maka

$$
f\left(\sum_{i=1}^{n} r_{i} x_{i}\right) \leq \sum_{i=1}^{n} r_{i} f\left(x_{i}\right)
$$

Ketaksamaan ini yang kemudian dikenal sebagai ketaksamaan umum Jensen.

Teorema[5] Fungsi f: $\mathrm{I}=[\mathrm{a}, \mathrm{b}] \subseteq \mathbb{R} \rightarrow \mathbb{R}$ dikatakan konveks jika dan hanya jika untuk sebarang $\mathrm{x}, \mathrm{w}, \mathrm{y} \in \mathrm{I}$ yang bersifat $\mathrm{x}<\mathrm{w}<\mathrm{y}$ berlaku

$$
\frac{f(w)-f(x)}{w-x} \leq \frac{f(y)-f(x)}{y-x} \leq \frac{f(y)-f(w)}{y-w}
$$

Teorema[5] Sebarang fungsi konveks adalah fungsi kontinu.

Definisi[4] Misal $\mathrm{f}$ adalah fungsi yang bernilai positif, f dikatakan log konveks jika fungsi $\log f(x)$ adalah konveks di daerah asal.

Definisi[6] Fungsi f: $\mathrm{I} \subset(0, \infty) \rightarrow \mathbb{R}$ dikatakan p-konveks, dengan $\mathrm{p}>0$, jika berlaku

$$
f\left(\left[t x^{p}+(1-t) y^{p}\right]^{1 / p}\right) \leq t f(x)+(1-t) f(y)
$$

Definisi[7] Untuk $0<\mathrm{x}<\infty$, didefinisikan fungsi gamma sebagai :

$$
\Gamma(x)=\int_{0}^{\infty} t^{x-1} e^{-t} d t
$$

Nilai integral konvergen untuk setiap x.

Teorema[8] Misalkan $\mathrm{f}, \mathrm{g}:[\mathrm{a}, \mathrm{b}] \rightarrow \mathbb{R}$ terintegralkan di $[\mathrm{a}, \mathrm{b}]$, dan $\mathrm{p}, \mathrm{q}>1$ bersifat $\frac{1}{\mathrm{p}}+$ $\frac{1}{\mathrm{q}}=1$ maka berlaku :

$$
\int_{a}^{b}|f(x) g(x)| d x \leq\left(\int_{a}^{b}|f(x)|^{p}\right)^{\frac{1}{p}}\left(\int_{a}^{b}|g(x)|^{q}\right)^{\frac{1}{q}}
$$




\section{Hasil dan Pembahasan}

Teorema. Misalkan $\Gamma(\mathrm{x})=\int_{0}^{\infty} \mathrm{t}^{\mathrm{x}-1} \mathrm{e}^{-\mathrm{t}} \mathrm{dt}, 0<\mathrm{x}<\infty$, maka $\Gamma$ memiliki sifat berikut :

i. $\quad \quad \quad \quad(x+1)=x \Gamma(x), 0<x<\infty$

ii. $\quad \Gamma(1)=1$

iii. $\log \Gamma$ konveks di $(0, \infty)$

Bukti :

i. $\quad \Gamma(x+1)=\int_{0}^{\infty} t^{(x+1)-1} e^{-t} d t$

$$
\begin{aligned}
& =\lim _{a \rightarrow \infty} \int_{0}^{a} t^{x} e^{-t} d t \\
& =\lim _{a \rightarrow \infty} \int_{0}^{a} t^{x} \frac{d\left(e^{-t}\right)}{-1} \\
& =\lim _{a \rightarrow \infty}\left[-\left.t^{x} e^{-t}\right|_{t=0} ^{t=a}-\int_{0}^{a} e^{-t} d\left(-t^{x}\right)\right] \\
& =\lim _{a \rightarrow \infty}\left[\left(-a^{x} e^{-a}-0\right)-\int_{0}^{a} e^{-t}(-x) t^{x-1} d t\right] \\
& =\lim _{a \rightarrow \infty} \int_{0}^{a} x t^{x-1} e^{-t} d t \\
& =x \Gamma(x)
\end{aligned}
$$

ii. $\quad \Gamma(1)=\lim _{a \rightarrow \infty} \int_{0}^{a} t^{1-1} e^{-t} d t$

$$
=\lim _{\mathrm{a} \rightarrow \infty} \int_{0}^{a} e^{-t} d t=\lim _{a \rightarrow \infty}-\left.e^{-t}\right|_{t=0} ^{t=a}=1
$$

iii. Dengan ketaksamaan Holder, akan diperoleh

$$
\begin{aligned}
& \left|\int_{0}^{\infty} f(s) g(s) h(s) d s\right| \leq\left(\int_{0}^{\infty}|f(s)|^{p} h(s) d s\right)^{\frac{1}{p}}\left(\int_{0}^{\infty}|g(s)|^{q} h(s) d s\right)^{\frac{1}{q}} \\
& \text { dengan , } q>1, \frac{1}{p}+\frac{1}{q}=1, h \geq 0 \text { di }(0, \infty) \text {, dan } f, g, h \text { terintegralkan di } \\
& (0, \infty) \text {. } \\
& \text { Misalkan } f(s)=s^{a(x-1)}, g(s)=s^{b(y-1)}, h(s)=e^{-s} \text { dengan } s \in(0, \infty) \text {, } \\
& x, y>0, a, b \geq 0 \text { dan } a+b=1 \text {. } \\
& \text { Dengan demikian didapat } \\
& \left|\int_{0}^{\infty} s^{a(x-1)} s^{b(y-1)} e^{-s} d s\right| \leq\left(\int_{0}^{\infty}\left|s^{a(x-1)}\right|^{\frac{1}{a}} e^{-s} d s\right)^{a}\left(\int_{0}^{\infty}\left|s^{b(y-1)}\right|^{\frac{1}{b}} e^{-s} d s\right)^{b}
\end{aligned}
$$




$$
\begin{gathered}
=\left(\int_{0}^{\infty} s^{x-1} e^{-s} d s\right)^{a}\left(\int_{0}^{\infty} s^{y-1} e^{-s} d s\right)^{b} \\
\Rightarrow \int_{0}^{\infty} s^{a x+b y-1} e^{-s} d s \leq\left(\int_{0}^{\infty} s^{x-1} e^{-s} d s\right)^{a}\left(\int_{0}^{\infty} s^{y-1} e^{-s} d s\right)^{b} \\
\Rightarrow \Gamma(a x+b y) \leq\left(\int_{0}^{\infty} s^{x-1} e^{-s} d s\right)^{a}\left(\int_{0}^{\infty} s^{y-1} e^{-s} d s\right)^{b}
\end{gathered}
$$

Dari sini diperoleh

$$
\begin{aligned}
\log \Gamma(a x+b y) & =\log \left(\int_{0}^{\infty} s^{a x+b y-1} e^{-s} d s\right) \\
\leq & \log \left(\left(\int_{0}^{\infty} s^{x-1} e^{-s} d s\right)^{a}\left(\int_{0}^{\infty} s^{y-1} e^{-s} d s\right)^{b}\right) \\
= & \log \left(\left(\int_{0}^{\infty} s^{x-1} e^{-s} d s\right)^{a}\right)+\log \left(\left(\int_{0}^{\infty} s^{y-1} e^{-s} d s\right)^{b}\right) \\
= & a \log \left(\int_{0}^{\infty} s^{x-1} e^{-s} d s\right)+b \log \left(\int_{0}^{\infty} s^{y-1} e^{-s} d s\right) \\
= & a \Gamma(x)+b \Gamma(y) .
\end{aligned}
$$

Sehingga dapat disimpulkan bahwa fungsi gamma adalah fungsi yang bersifat (i),(ii) dan (iii).

Teorema. Misalkan $\mathrm{f:}(0, \infty) \rightarrow(0, \infty)$ adalah fungsi yang bersifat sebagai berikut :

Bukti :

i. $\quad f(x+1)=x f(x), 0<x<\infty$

ii. $\quad f(1)=1$

iii. $\log f$ konveks di $(0, \infty)$

Karena $(x+1)=x f(x)$, maka untuk sebarang $n \in \mathbb{N}$ didapatkan

$$
f(x+n)=(x+n-1)(x+n-2)(x+n-3) \ldots(x+1) x f(x), 0<x<\infty .
$$

Karena $\mathrm{f}(1)=1$ maka dengan mudah akan terlihat bahwa $\mathrm{f}(\mathrm{n})=(\mathrm{n}-1) !, \forall \mathrm{n} \in \mathbb{N}$. Selanjutnya akan ditinjau nilai $\mathrm{f}(\mathrm{x})$ saat $0<\mathrm{x}<1$. Perhatikan bahwa $\forall \mathrm{n} \in$ $\mathbb{N}$ berlaku $n-1<n<n+x<n+1,0<x<1$.

Karena $\log \mathrm{f}$ konveks maka berdasarkan Teorema diperoleh ketaksamaan berikut 


$$
\begin{aligned}
& \frac{\log (f(n))-\log (f(n-1))}{n-(n-1)} \leq \frac{\log (f(n+x))-\log (f(n))}{n+x-n} \\
& \leq \frac{\log (f(n+1))-\log (f(n))}{(n+1)-n} \\
& \frac{\log ((n-1) !)-\log ((n-2) !)}{1} \leq \frac{\log (f(n+x))-\log ((n-1) !)}{x} \\
& \leq \frac{\log (n !)-\log ((n-1) !)}{1} \\
& \log \left(\frac{(n-1) !}{(n-2) !}\right) \leq \frac{\log (f(n+x))-\log ((n-1) !)}{x} \leq \log \left(\frac{n !}{(n-1) !}\right) \\
& \log (n-1) \leq \frac{\log (f(n+x))-\log ((n-1) !)}{x} \leq \log (n) \\
& x \log (n-1) \leq \log (f(n+x))-\log ((n-1) !) \leq x \log (n) \\
& x \log (n-1)+\log ((n-1) !) \leq \log (f(n+x)) \leq x \log (n)+\log ((n-1) !) \\
& \log \left((n-1)^{x}(n-1) !\right) \leq \log (f(n+x)) \leq \log \left(n^{x}(n-1) !\right) \\
& (n-1)^{x}(n-1) ! \leq f(n+x) \leq n^{x}(n-1) ! \\
& (n-1)^{x}(n-1) ! \leq(x+n-1)(x+n-2)(x+n-3) \ldots(x+1) x f(x) \\
& \leq n^{x}(n-1) \text { ! } \\
& \frac{(n-1)^{x}(n-1) !}{(x+n-1)(x+n-2) \ldots(x+1) x} \leq f(x) \leq \frac{n^{x}(n-1) !}{(x+n-1)(x+n-2) \ldots(x+1) x} \\
& \frac{(n-1)^{x}(n-1) !}{(x+n-1)(x+n-2) \ldots(x+1) x} \leq f(x) \\
& \leq \frac{n^{x} n !}{(x+n)(x+n-1) \ldots(x+1) x}\left(\frac{x+n}{n}\right)
\end{aligned}
$$

Ketaksamaan ini berlaku $\forall \mathrm{n} \in \mathbb{N}$, sehingga dengan memilih $\mathrm{n}+1$ pada ruas kiri didapat:

$$
\frac{n^{x} n !}{(x+n)(x+n-1) \ldots(x+1) x} \leq f(x) \leq \frac{n^{x} n !}{(x+n)(x+n-1) \ldots(x+1) x}\left(\frac{x+n}{n}\right) .
$$

Karena ketaksamaan ini berlaku $\forall \mathrm{n} \in \mathbb{N}$, maka dengan mengambil nilai $\mathrm{n}$ yang sangat besar sehingga $\lim _{n \rightarrow \infty}\left(\frac{x+n}{n}\right)=1$ berakibat ruas kanan dan ruas kiri ketaksamaan tersebut memiliki nilai yang sama. Sehingga diperoleh nilai $\mathrm{f}(\mathrm{x})$ yaitu

$$
f(x)=\lim _{n \rightarrow \infty} \frac{n^{x} n !}{(x+n)(x+n-1) \ldots(x+1) x}
$$

Teorema. Misalkan $\mathrm{f}(\mathrm{x})=\lim _{\mathrm{n} \rightarrow \infty} \frac{\mathrm{n}^{\mathrm{x}} \mathrm{n} !}{(\mathrm{x}+\mathrm{n})(\mathrm{x}+\mathrm{n}-1) \ldots(\mathrm{x}+1) \mathrm{x}}$ dengan $0<\mathrm{x}<\infty$ maka $\mathrm{f}(\mathrm{x})=$ $\Gamma(x)$. 
Bukti :

Misalkan $\quad \Gamma_{\mathrm{m}}(\mathrm{x})=\frac{\mathrm{m}^{\mathrm{x}} \mathrm{m} !}{(\mathrm{x}+\mathrm{m})(\mathrm{x}+\mathrm{m}-1) \ldots(\mathrm{x}+1) \mathrm{x}}, \quad$ akan dibuktikan bahwa $\Gamma_{\mathrm{m}}(\mathrm{x})$ ekivalent dengan :

$$
\int_{0}^{1} m^{x}(1-t)^{m} t^{x-1} d t
$$

Untuk membuktikan hal tersebut, akan digunakan induksi matematika

Untuk $\mathrm{m}=1$ didapat

$$
\begin{array}{rl}
\int_{0}^{1} 1^{x}(1-t)^{1} t^{x-1} & d t=\int_{0}^{1}(1-t) t^{x-1} d t \\
& =\int_{0}^{1} t^{x-1} d t-\int_{0}^{1} t^{x} d t \\
& =\left[\frac{t^{x}}{x}-\frac{t^{x+1}}{x+1}\right]_{0}^{1} \\
& =\frac{1}{x}-\frac{1}{x+1} \\
& =\frac{1}{x(x+1)} \\
& =\Gamma_{1}(x)
\end{array}
$$

Misalkan untuk $\mathrm{m}=\mathrm{k}$ benar yaitu

Untuk $\mathrm{m}=\mathrm{k}+1$ diperoleh

$$
\int_{0}^{1} k^{x}(1-t)^{k} t^{x-1} d t=\Gamma_{k}(x)
$$

$$
\int_{0}^{1}(k+1)^{x}(1-t)^{k+1} t^{x-1} d t=(k+1)^{x}\left[\int_{0}^{1}(1-t)^{k+1} t^{x-1} d t\right]
$$

Misalkan $\mathrm{u}=(1-\mathrm{t})^{\mathrm{k}+1}$ dan $\mathrm{v}=\frac{\mathrm{t}^{\mathrm{x}}}{\mathrm{x}}$, maka dengan metode integral parsial diperoleh

$$
\begin{aligned}
\int_{0}^{1} & (k+1)^{x}(1-t)^{k+1} t^{x-1} d t \\
& =(k+1)^{x}\left[\int_{0}^{1}(1-t)^{k+1} t^{x-1} d t\right] \\
& =(k+1)^{x}\left[\left.u v\right|_{0} ^{1}-\int_{0}^{1} v d u\right]
\end{aligned}
$$




$$
\begin{aligned}
& =(k+1)^{x}\left[\left.(1-t)^{k+1} \frac{t^{x}}{x}\right|_{0} ^{1}-\int_{0}^{1} \frac{t^{x}}{x}(-(k+1))(1-t)^{k} d t\right] \\
& =\frac{(k+1)^{x+1}}{x} \int_{0}^{1} t^{x}(1-t)^{k} d t \\
& =\frac{(k+1)^{x+1}}{x} \frac{\Gamma_{k}(x+1)}{k^{x+1}} \\
& =\frac{(k+1)^{x+1}}{x} \frac{k^{x+1} k !}{(x+k+1)(x+k) \ldots(x+2)(x+1) k^{x+1}} \\
& =\frac{(k+1)^{x+1} k !}{(x+k+1)(x+k) \ldots(x+2)(x+1) x} \\
& =\frac{(k+1)^{x}(k+1) !}{(x+k+1)(x+k) \ldots(x+2)(x+1) x} \\
& =\Gamma_{k+1}(x) \quad
\end{aligned}
$$

Dengan demikian terbukti bahwa

$$
\frac{m^{x} m !}{(x+m)(x+m-1) \ldots(x+1) x}=\int_{0}^{1} m^{x}(1-t)^{m} t^{x-1} d t,
$$

Dengan subtitusi $\mathrm{t}=\frac{\mathrm{s}}{\mathrm{m}}$ mengakibatkan

$$
\begin{aligned}
d s=m d t & ,(1-t)^{m}=\left(1-\frac{s}{m}\right)^{m}, m^{x-1} t^{x-1}=s^{x-1} \\
& \int_{0}^{1} m^{x}(1-t)^{m} t^{x-1} d t=\int_{0}^{m}\left(1-\frac{s}{m}\right)^{m} s^{x-1} d s
\end{aligned}
$$

Dari sini diperoleh

$$
\Gamma_{m}(x)=\int_{0}^{m}\left(1-\frac{s}{m}\right)^{m} s^{x-1} d s
$$

Selanjutnya akan dibuktikan bahwa untuk $\mathrm{m} \geq 2$ dan $0 \leq \mathrm{t} \leq \mathrm{m}$ berlaku

$$
0 \leq e^{-t}-\left(1-\frac{t}{m}\right)^{m} \leq e^{-t} \frac{t^{2}}{n}
$$

Untuk m yang sangat besar, akan dipunyai $\left(1-\frac{\mathrm{t}}{\mathrm{m}}\right)^{\mathrm{m}} \leq \mathrm{e}^{-\mathrm{t}}$ dan $\left(1+\frac{\mathrm{t}}{\mathrm{m}}\right)^{\mathrm{m}} \leq \mathrm{e}^{\mathrm{t}}$

Berdasarkan hal tersebut diperoleh

$$
\begin{aligned}
& e^{t}\left(1-\frac{t}{m}\right)^{m} \leq e^{t} e^{-t}=1 \text { dan }\left(1-\frac{t^{2}}{m}\right) \leq\left(1-\frac{t^{2}}{m^{2}}\right)^{m} \leq\left(1-\frac{t}{m}\right)^{m}(1+ \\
& \left.\frac{t}{m}\right)^{m} \leq e^{t}\left(1-\frac{t}{m}\right)^{m}
\end{aligned}
$$


Akibatnya didapat $\left(1-\frac{\mathrm{t}^{2}}{\mathrm{~m}}\right) \leq \mathrm{e}^{\mathrm{t}}\left(1-\frac{\mathrm{t}}{\mathrm{m}}\right)^{\mathrm{m}} \leq 1$ yang ekivalent dengan $0 \leq$ $e^{-t}-\left(1-\frac{t}{m}\right)^{m} \leq e^{-t} \frac{t^{2}}{m}$

Dari sini diperoleh

$$
\begin{aligned}
& 0 \leq \Gamma(x)-\Gamma_{m}(x)=\lim _{m \rightarrow \infty}\left\{\int_{0}^{m} e^{-t} t^{x-1} d t-\int_{0}^{m}\left(1-\frac{t}{m}\right)^{m} t^{x-1} d t\right\} \\
&= \lim _{m \rightarrow \infty}\left\{\int_{0}^{m} t^{x-1}\left(e^{-t}-\left(\left(1-\frac{t}{m}\right)^{m}\right)\right) d t\right\} \\
& \leq \lim _{m \rightarrow \infty} \int_{0}^{m} t^{x-1} e^{-t} \frac{t^{2}}{m} d t \\
& \leq \lim _{m \rightarrow \infty} \frac{\Gamma(x+2)}{m}=0 . \\
& \Gamma(x)=\Gamma_{m}(x)
\end{aligned}
$$

Berdasarkan teorema-teorema di atas, terlihat bahwa fungsi gamma adalah satu-satunya fungsi di bilangan positif yang bersifat (i), (ii) dan (iii).

Teorema. Misalkan $\mathrm{f}: \mathrm{I} \subset(0, \infty) \rightarrow \mathbb{R}$ adalah fungsi $\mathrm{p}$-konveks dengan $\mathrm{p}>0$ dan $\mathrm{a}, \mathrm{b} \in$ I. Maka ketaksamaan berikut berlaku pada $\mathrm{f}$

Bukti :

$$
f\left(\left[\frac{a^{p}+b^{p}}{2}\right]^{\frac{1}{p}}\right) \leq \frac{p}{b^{p}-a^{p}} \int_{0}^{1} \frac{f(x)}{x^{1-p}} d x \leq \frac{f(a)+f(b)}{2}
$$

Berdasarkan definisi, maka $\mathrm{f}: \mathrm{I} \subset(0, \infty) \rightarrow \mathbb{R}$ dan $\mathrm{p}>0$ dikatakan p-konveks jika berlaku

$$
f\left(\left[t x^{p}+(1-t) y^{p}\right]^{1 / p}\right) \leq t f(x)+(1-t) f(y), \quad t \in[0,1], \quad x, y \in I
$$

Misalkan $\mathrm{f}: \mathrm{I}=[\mathrm{a}, \mathrm{b}] \subset(0, \infty) \rightarrow \mathbb{R}$ adalah fungsi p-konveks dengan $\mathrm{a}, \mathrm{b} \in$ $(0, \infty)$ dan $\mathrm{p}>0$, maka dengan mengambil $\mathrm{t}=\frac{1}{2}$ didapat

$$
f\left(\left[\frac{x^{p}+y^{p}}{2}\right]^{\frac{1}{p}}\right) \leq \frac{f(x)+f(y)}{2}, \forall x, y \in I
$$


Pilih $\mathrm{x}=\left[\mathrm{ta}^{\mathrm{p}}+(1-\mathrm{t}) \mathrm{b}^{\mathrm{p}}\right]^{\frac{1}{\mathrm{p}}}$ dan $\mathrm{y}=\left[\mathrm{tb}^{\mathrm{p}}+(1-\mathrm{t}) \mathrm{a}^{\mathrm{p}}\right]^{\frac{1}{\mathrm{p}}}$, untuk suatu $\mathrm{t} \in[0,1]$. Jelas bahwa , $y \in$ I. Sehingga didapat

$$
f\left(\left[\frac{a^{p}+b^{p}}{2}\right]^{\frac{1}{p}}\right) \leq \frac{f\left(\left[t a^{p}+(1-t) b^{p}\right]^{\frac{1}{p}}\right)+f\left(\left[t b^{p}+(1-t) a^{p}\right]^{\frac{1}{p}}\right)}{2}
$$

Dengan mengintegralkan kedua ruas terhadap t dengan interval $[0,1]$ didapat

$$
\begin{aligned}
& \int_{0}^{1} f\left(\left[\frac{a^{p}+b^{p}}{2}\right]^{\frac{1}{p}}\right) d t \leq \int_{0}^{1}\left[\frac{f\left(\left[t a^{p}+(1-t) b^{p}\right]^{\frac{1}{p}}\right)+f\left(\left[t b^{p}+(1-t) a^{p}\right]^{\frac{1}{p}}\right)}{2}\right] d t \\
& f\left(\left[\frac{a^{p}+b^{p}}{2}\right]^{\frac{1}{p}}\right) \leq \frac{\int_{0}^{1} f\left(\left[t a^{p}+(1-t) b^{p}\right]^{\frac{1}{p}}\right) d t+\int_{0}^{1} f\left(\left[t b^{p}+(1-t) a^{p}\right]^{\frac{1}{p}}\right) d t}{2}
\end{aligned}
$$

Dengan metode subtitusi, akan didapat

$$
f\left(\left[\frac{a^{p}+b^{p}}{2}\right]^{\frac{1}{p}}\right) \leq \frac{\frac{p}{a^{p}-b^{p}} \int_{b}^{a} \frac{f(x)}{x^{1-p}} d x+\frac{p}{b^{p}-a^{p}} \int_{a}^{b} \frac{f(x)}{x^{1-p}} d x}{2}=\frac{p}{b^{p}-a^{p}} \int_{a}^{b} \frac{f(x)}{x^{1-p}} d x
$$

Disisi lain, kita punyai

$$
\frac{p}{b^{p}-a^{p}} \int_{a}^{b} \frac{f(x)}{x^{1-p}} d x=\frac{\int_{0}^{1} f\left(\left[t a^{p}+(1-t) b^{p}\right]^{\frac{1}{p}}\right) d t+\int_{0}^{1} f\left(\left[t b^{p}+(1-t) a^{p}\right]^{\frac{1}{p}}\right) d t}{2}
$$

Karena f adalah fungsi $\mathrm{p}$-konveks maka berlaku

Dari sini diperoleh

$$
\begin{aligned}
& f\left(\left[t a^{p}+(1-t) b^{p}\right]^{\frac{1}{p}}\right) \leq t f(a)+(1-t) f(b) \\
& f\left(\left[t b^{p}+(1-t) a^{p}\right]^{\frac{1}{p}}\right) \leq t f(b)+(1-t) f(a)
\end{aligned}
$$

$$
\begin{gathered}
\frac{p}{b^{p}-a^{p}} \int_{a}^{b} \frac{f(x)}{x^{1-p}} d x=\frac{\int_{0}^{1} f\left(\left[t a^{p}+(1-t) b^{p}\right]^{\frac{1}{p}}\right) d t+\int_{0}^{1} f\left(\left[t b^{p}+(1-t) a^{p}\right]^{\frac{1}{p}}\right) d t}{2} \\
\leq \frac{\int_{0}^{1}[t f(a)+(1-t) f(b)] d t+\int_{0}^{1}[t f(b)+(1-t) f(a)] d t}{2}
\end{gathered}
$$




$$
\begin{aligned}
& =\int_{0}^{1} \frac{[t f(a)+(1-t) f(b)+t f(b)+(1-t) f(a)]}{2} \\
& =\int_{0}^{1} \frac{f(a)+f(b)}{2} \\
& =\frac{f(a)+f(b)}{2} \\
& f\left(\left[\frac{a^{p}+b^{p}}{2}\right]^{\frac{1}{p}}\right) \leq \frac{p}{b^{p}-a^{p}} \int_{0}^{1} \frac{f(x)}{x^{1-p}} d x \leq \frac{f(a)+f(b)}{2}
\end{aligned}
$$

\section{Kesimpulan}

Berdasarkan hasil dan pembahasan, diperoleh kesimpulan sebagai berikut :

1. Fungsi gamma adalah satu-satunya fungsi di bilangan positif yang memiliki sifat sifat yaitu

i. $\quad \quad \quad \quad(x+1)=x \Gamma(x), 0<x<\infty$

ii. $\quad \Gamma(1)=1$

iii. $\quad \log \Gamma$ konveks di $(0, \infty)$

2. Untuk $p>0$ dan $f: I \subset(0, \infty) \rightarrow \mathbb{R}$ maka ketaksamaan Hadamard pada $\mathrm{f}$ adalah

$$
f\left(\left[\frac{a^{p}+b^{p}}{2}\right]^{\frac{1}{p}}\right) \leq \frac{p}{b^{p}-a^{p}} \int_{0}^{1} \frac{f(x)}{x^{1-p}} d x \leq \frac{f(a)+f(b)}{2}
$$

\section{Referensi}

[1] Pachpatte, 2005, Mathematical Inequalities, Elsevier, Inc., USA.

[2] Gunawan, H., 2016, Pengantar Analisis Real, Institut Teknologi Bandung, Bandung.

[3] Abramowitz, M., dan Stegun, I.A., 1972, Handbook of Mathematical Functions with Formulas, Graph, and Mathematical Tables, United States Department of Commerce, USA.

[4] Iscan, I., dan Kunt, M.,2016, Hermite-Hadamard-Fejer type Inequalities for pconvex Functions, International Journal Analisis Applications Vol.23 Hal 215-230, Turkey.

[5] Artin, E., 1964, The Gamma Function, Holt Rinehart and Winston, Inc., New York.

[6] Rudin, W., 1976, Principles of Mathematical Analysis, McGraw-Hill, Inc., New York. 
[7] Kreyszig, E., 1978, Introductory Functional Analysis with Applications, John Wiley and Sons, Inc., Canada.

[8] Bartle, R.G., dan Sherbert, D.R., 2011, Introduction To Real Analysis Fourth Edition, John Wiley and Sons, Inc., Illinois.

[9] Noor, M.I., dkk , 2016, Hermite-Hadamard Inequalities for Differentiable p-convex Functions using Hypergeometric Functions, Publications de 1 Institut Mathematique Vol.100 Hal.251-257.

[10] Stewart, J., 2008, Calculus Early Transcendentals Sixth Edition, Thomson Learning, Inc., USA.

[11] Wu, S., dan Chen, F., 2014, Fejer and Hermite-Hadamard type Inequalities for Harmonically Convex Functions, Journal Applications Mathematic Vol 2014 Article id : 386806. 